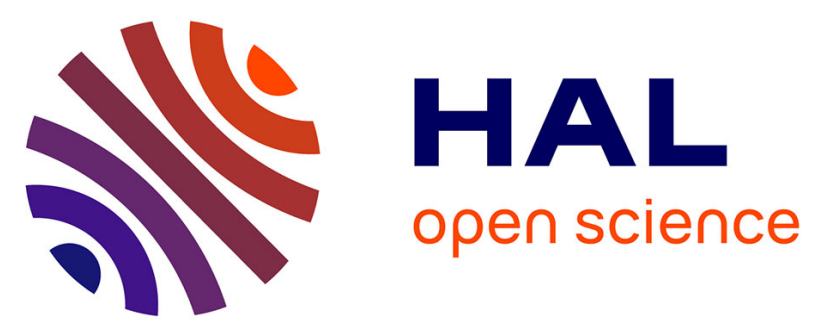

\title{
A stable marriage framework for distributed virtual MIMO coalition formation
}

\author{
Rodrigo-Alberto Vaca-Ramirez, Eitan Altman, John S. Thompson,
} Victor-Manuel Ramos-Ramos

\section{To cite this version:}

Rodrigo-Alberto Vaca-Ramirez, Eitan Altman, John S. Thompson, Victor-Manuel Ramos-Ramos. A stable marriage framework for distributed virtual MIMO coalition formation. IEEE PIMRC - 24th International Symposium on Personal Indoor and Mobile Radio Communications, Sep 2013, London, United Kingdom. pp.2707-2712, 10.1109/PIMRC.2013.6666606 . hal-00926597

\section{HAL Id: hal-00926597 https://hal.inria.fr/hal-00926597}

Submitted on 9 Jan 2014

HAL is a multi-disciplinary open access archive for the deposit and dissemination of scientific research documents, whether they are published or not. The documents may come from teaching and research institutions in France or abroad, or from public or private research centers.
L'archive ouverte pluridisciplinaire HAL, est destinée au dépôt et à la diffusion de documents scientifiques de niveau recherche, publiés ou non, émanant des établissements d'enseignement et de recherche français ou étrangers, des laboratoires publics ou privés. 


\title{
A Stable Marriage Framework for Distributed Virtual MIMO Coalition Formation
}

\author{
Rodrigo A. Vaca Ramírez ${ }^{(1)}$, Eitan Altman $^{(2)}$, John S. Thompson ${ }^{(1)}$, and Víctor M. Ramos R. ${ }^{(3)}$ \\ (1) The University of Edinburgh, Institute for Digital Communications \\ King's Buildings, Mayfield Road, Edinburgh, EH9 3JL, UK \\ ${ }^{(2)}$ INRIA, Sophia Antipolis Cedex, BP93, 06902 France \\ (3) Universidad Autónoma Metropolitana (UAM) \\ Department of Electrical Engineering Iztapalapa, Mexico City \\ E-Mail: \{r.vaca, john.thompson\}@ed.ac.uk, eitan.altman@inria.fr,vicman@xanum.uam.mx
}

\begin{abstract}
In this paper, a distributed algorithm for energy efficient virtual Multiple-input Multiple-output (MIMO) coalition formation is proposed. We model cooperation as a game theoretic approach derived from the concept of stable marriage with incomplete lists (SMI). Thus, single antenna devices such as mobile and relay stations are allowed to cooperate in order to improve the user's and system's energy efficiency. Our approach is focused on optimizing the circuit consumed power of the single antenna devices rather than on the transmitted power, thus the total device power consumption including the effect of the power amplifiers is taken into account. Furthermore, we show analytically and by simulation that under certain conditions cooperation does not improve the energy efficiency metric of network users, thus single antenna devices prefer to transmit independently in order to maintain the user's performance in the network. Additionally, our distributed approach reduces the communication overhead by $76 \%$ when compared with a centralized global optimum scheme, which is coordinated from the base station.
\end{abstract}

\section{INTRODUCTION}

Multi-antenna systems have emerged as an effective way to improve capacity and energy efficiency in wireless networks. It has been shown in [1] that Multiple-input Multiple-output (MIMO) systems require less transmitted power to achieve the same rate requirements than single antenna systems. In fourth generation (4G) networks such as Long Term Evolution (LTE), a base station (BS) can be equipped with multiple antennas. Nevertheless, mobile stations (MSs) may not support more than one single antenna due to physical constraints $[2,3]$. Fortunately, if we allow individual single antenna devices such as mobile and relay stations to cooperate with each other on information transmission, a virtual MIMO link can be constructed $[1,2]$.

Cooperation in virtual MIMO systems allows a wireless user to obtain important gains in spectral and energy efficiency. However, the design of an efficient cooperation algorithm faces various challenges. On one hand, cooperation costs such as power expenditure can limit the benefits or even degrade the user's performance. On the other hand, if cooperation between wireless nodes is coordinated by a centralized entity, an extra signaling overhead is required for information exchange to set up and maintain the cooperative link. Thus, the design of a distributed algorithm that allows the single antenna devices to autonomously decide "when to cooperate" and "with whom to cooperate" is highly challenging but desirable in practice [4].

Energy efficiency has become an important research topic for mobile stations due to their limited battery resources. Moreover, in mobile stations most of the power expenditure is due to the radio frequency (RF) circuits [5]. However, current research in energy efficiency has been mainly focused on reducing the transmitted power rather than on reducing the total consumed power of the MS [6]. Hence, taking into consideration the power amplifiers' effect for reducing the total consumed power is crucial for improving the MS's battery life, and maintaining the overall MS performance in the network [7].

The main contribution of this paper is to provide a distributed coalition formation framework, which allows single antenna devices to cooperate and form a virtual MIMO link, in order to reduce the energy consumption in the network. Additionally, it is shown analytically and by simulation that under certain conditions forming a virtual MIMO channel does not provide an energy efficient solution, when the circuit power consumption is considered.

The remainder of this paper is as follows, in Section II we present the problem scenario and performance metrics. In Section III, we describe our cooperative framework. A summary of the comparison schemes and the simulation scenario are described in Section IV. Results are presented in Section V. Finally, Section VI offers concluding remarks.

\section{System Scenario And Performance Metrics}

In this work, we consider $N$ mobile stations (MSs), all of them equipped with a single antenna sending data on the uplink to a multiple antenna base station (BS). Additionally, $R$ single antenna relay stations (RSs) are uniformly distributed through the cell, assuming $R \gg N$. When cooperating, the single antenna devices (MSs and RSs) form a virtual $2 \times M_{r}$ MIMO link, where $M_{r}$ is the number of antennas at the BS side. If cooperation is not possible, the MSs will transmit to the BS on their own in (Single-input Multiple-output) SIMO mode.

The system bandwidth $B(\mathrm{~Hz})$ is divided into $X$ resource blocks (RBs). Hence, an orthogonal frequency division multi- 


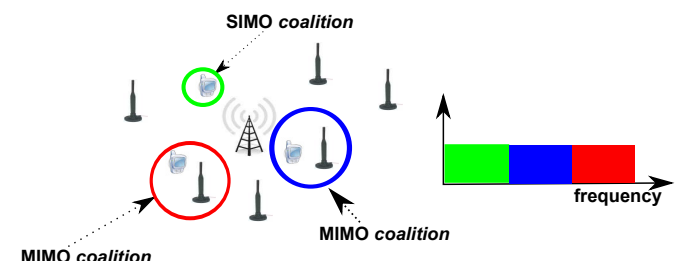

Fig. 1: User cooperation example coalitions considering an OFDMA transmission model.

ple access (OFDMA) system is constructed, where each RB is assigned to each coalition independently to avoid co-channel interference (CCI). In this paper the term coalition refers to a single user MIMO or SIMO respectively. In Fig. 1, we show an illustration of the system scenario.

\section{A. Channel Model}

1) Reverse Link: The uplink received signal at the base station (BS) separated by a distance $d_{k}$ from a coalition $k$ is determined by path loss, log-normal shadowing, and channel variations caused by fading. We assume a flat fading Rayleigh channel, thus the fading coefficients of a $2 \times M_{r}$ MIMO channel may be represented by a matrix

$$
\mathbf{H}=\left[\begin{array}{cc}
h_{1,1} & h_{1,2} \\
h_{2,1} & h_{2,2} \\
\vdots & \vdots \\
h_{M_{r}, 1} & h_{M_{r}, 2}
\end{array}\right],
$$

where each entry is a Zero Mean Circulant Symmetric Complex Gaussian (ZMCSCG) random variable with unit variance [1]. SIMO systems can be treated as a special case of MIMO, where the channel is represented by a vector $\mathbf{h}=\left[h_{1}, h_{2}, \ldots, h_{M_{r}}\right]^{T}$. In addition, path loss and shadowing are modeled as a power fall off that further attenuates the transmitted signal, hence the average power of the received signal $P_{r}$ can be expressed as follows [6]:

$$
P_{r}=P_{t} 10^{\frac{-L\left(d_{k}\right)+X_{\sigma}}{10}},
$$

where $P_{t}$ represents the power of the transmitted signal, $X_{\sigma}$ is the log-normal shadowing value $(\mathrm{dB})$ with standard deviation $\sigma$, and $L\left(d_{k}\right)$ is the distance-dependent path loss (dB), which is calculated as follows:

$$
L\left(d_{k}\right)=15.3+37.6 \log _{10}\left(d_{k}\right) .
$$

When a MIMO coalition is formed, cooperation is made using a short range transmitter, hence it can be assumed that the single antenna devices are close enough to experience the same channel statistics, thus shadowing and path loss remain the same for the devices forming a virtual MIMO link.

The channel is assumed to be known at the transmitter and receiver. Thus, the received signal at the BS may be expressed as

$$
\mathbf{y}_{k}=\sqrt{\frac{P_{r}}{M_{t}}} \mathbf{H w} s+\mathbf{n},
$$

where $\mathbf{y}_{k}$ is the received signal at the BS from the $k$-th MIMO coalition, $M_{t}$ is the number of transmitting antennas per coalition, $s$ is the information symbol with unit energy, $\mathbf{n}$ is the noise, and $\mathbf{w}$ is a complex weight vector, that must satisfy $\|\mathbf{w}\|_{F}^{2}=M_{t}$ to constrain the total average transmitted power, where $\|\cdot\|_{F}^{2}$ is the Frobenius norm. It should be noted that for a SIMO coalition Eq. (4) becomes: $\mathbf{y}_{k}=\sqrt{P_{r}} \mathbf{h} s+\mathbf{n}$. Furthermore, the signal-to-noise ratio (SNR) for a MIMO coalition is given by [1]:

$$
\eta_{k \_m i m o}=\frac{\left\|\mathbf{g}^{H} \mathbf{H w}\right\|_{F}^{2} P_{r}}{M_{t}\|\mathbf{g}\|_{F}^{2} \rho},
$$

where $\mathbf{g}$ is an $M_{r} \times 1$ vector of complex weights which multiplies $\mathbf{y}_{k}$ at the $\mathrm{BS}$ side and $\rho$ is the noise power. Hence, maximizing the SNR at the receiver is equivalent to maximizing $\left\|\mathbf{g}^{H} \mathbf{H w}\right\|_{F}^{2} /\|\mathbf{g}\|_{F}^{2}$. By using singular value decomposition (SVD) the channel matrix can be represented as $\mathbf{H}=\mathbf{U} \Sigma \mathbf{V}^{H}$, where the columns of $\mathbf{V}$ and $\mathbf{U}$ are known as the input and output singular vectors respectively, and $\Sigma=\operatorname{diag}\left\{\sigma_{1}, \sigma_{2}, \ldots, \sigma_{j}\right\}$ with $\sigma_{i} \geq 0$, where $\sigma_{i}$ is the $i$-th singular value of the channel, and $j$ is the rank of $\mathbf{H}$. Thus, the right choice of $\mathbf{w} / \sqrt{M_{t}}$ and $\mathrm{g}$ that maximizes the SNR are the corresponding input and output singular value vectors corresponding to the maximum singular value $\sigma_{\max }$ of $\mathbf{H}$ [1]. Therefore, the received SNR at the BS side from the $k$-th MIMO coalition may be expressed as follows:

$$
\eta_{k_{-} \text {mimo }}=\frac{\sigma_{\max }^{2} P_{r}}{\rho},
$$

In the case of a SIMO coalition, the received SNR at the BS may be expressed by [1]:

$$
\eta_{k_{-} \text {simo }}=\frac{\|\mathbf{h}\|_{F}^{2} P_{r}}{\rho},
$$

2) Cooperative Link: If cooperation is achieved, the cooperative link between the MS- $n$ and the RS- $r$ link is modeled as following $\kappa^{t h}$-power path loss (loss $\approx \frac{1}{d_{n r}^{\kappa}}$ ) with Additive White Gaussian Noise (AWGN). Hence, the received power $P_{n r}$ from the signal transmitted from the MS- $n$ to the RS- $r$ is expressed as follows:

$$
P_{n r}=\frac{P_{\mathrm{tcop}}}{d_{n r}^{\kappa}}
$$

where $P_{\text {tcop }}$ is the transmitted power for cooperation and $d_{n r}$ is the distance between the MS- $n$ and the RS- $r$. Hence, the signal-noise ratio (SNR) at the RS side is given by

$$
\eta_{n r}=\frac{P_{n r}}{\rho}
$$

3) Virtual MIMO Link: In Fig. 2 a virtual $2 \times M_{r}$ MIMO link is shown. The MS forwards the information symbol $s$ to the RS using the cooperative (short range) link. Furthermore, in the reverse link the MS and RS transmit the symbol $s$ to the BS through the MIMO channel $\mathbf{H}$. The reverse and cooperative link are designed orthogonal to each other, in order to prevent mutual interference. 


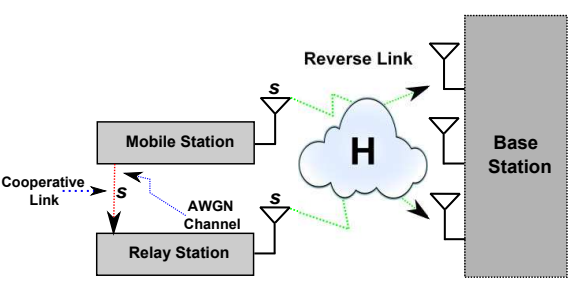

Fig. 2: A Virtual $2 \times M_{r}$ MIMO link.

\section{B. Physical Components Power Consumption Model}

In this paper, we consider the circuit power consumption of the MS's and RS's components, which is due to information transmission in the reverse and the cooperative link. The reverse link power consumption is dependent of components such as the baseband (BB) module and the radio frequency (RF) module. The former comprises the power consumed for the baseband signal processing (e.g., channel coding and decoding), and the latter incorporates the power consumption of the radio frequency components (e.g., the power amplifier). Hence, in order to model the power expenditure of both modules, we adopt the model presented in [5], where the authors make an analysis of the power consumption of both modules for a Long Term Evolution (LTE) MS. Hence, the total power consumed in SIMO transmission mode $P_{\text {tot_simo }}$ only depends on the transmitted power in the uplink $P_{t}$. Additionally, the total consumed power to form a virtual MIMO link $P_{\text {tot_mimo }}$ is a function of the transmitted power in the cooperative link $P_{\text {tcop }}$, the transmitted power in the uplink $P_{t}$, and the distribution of the transmitted power between the MS and the RS, which is due to the weight vector w. Hence, the total consumed power for a SIMO and MIMO coalition is modeled by

$$
\begin{gathered}
P_{\text {tot_simo }}\left(P_{t}\right)=P_{\text {circ }}\left(P_{t}\right) \\
P_{\text {tot_mimo }}\left(P_{t}, P_{\text {tcop }}\right)=P_{\text {circ }}\left(\frac{P_{t}\left\|w_{\mathrm{ms}}\right\|^{2}}{M_{t}}\right)+ \\
P_{\text {circop }}\left(P_{\text {tcop }}\right)+P_{\text {circ }}\left(\frac{P_{t}\left\|w_{\mathrm{rs}}\right\|^{2}}{M_{t}}\right)
\end{gathered}
$$

where $P_{\text {circ }}$ is the circuit power consumed in the reverse link, $P_{\text {circop }}$ is the circuit consumed power in the short range link (cooperative link), and $w_{\mathrm{ms}}$ and $w_{\mathrm{rs}}$ are the elements of the weight vector $\mathbf{w}$ for the MS and RS respectively in a $2 \times M_{r}$ MIMO channel.

Furthermore, a power amplifier array is assumed to model the circuit consumed power of the RF module [5,7], as presented in Fig 3. The power amplifier array is constructed based on a low power amplifier (LPA) and two high power amplifiers HPA 1, and HPA 2, where the power amplifier efficiency is assumed equal for both high power amplifiers; however HPA 2 is designed to transmit up to the half of the maximum transmitted power of HPA 1 . Therefore, the circuit

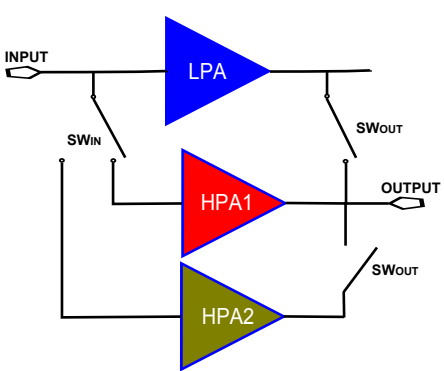

Fig. 3: Internal model of the power amplifier for the RF module.

power consumption in the uplink $P_{\text {circ }}[\mathrm{W}]$ is obtained by:

$$
P_{\text {circ }}\left(P_{t}^{*}\right)= \begin{cases}2+0.005\left(P_{t}^{*}\right)-A & 10 \geq P_{t}^{*} \\ \frac{1.2+0.12\left(P_{t}^{*}\right)-\left(A-P_{B B}\right)}{2} & 20 \geq P_{t}^{*}>10 \\ 1.2+0.12\left(P_{t}^{*}\right)-A & 23 \geq P_{t}^{*}>20\end{cases}
$$

where the $P_{t}^{*}[\mathrm{dBm}]$ is the input value of $P_{\text {circ }}$ in Equations (10) and (11), and $A$ is a set of constant values defined as follows [5]:

$$
A=P_{T x}+P_{\text {con }}-P_{B B}[W],
$$

where $P_{T x}$ is the base power that the RF chain consumes in transmission mode, $P_{\text {con }}$ is the MS's power consumption when connected to the BS, and $P_{B B}$ is the power consumed by the BB module [5].

As we have stated, the cooperative link is a short range communications link, thus in order to model its circuit power consumption $P_{\text {circop }}$, we use the LPA model:

$P_{\text {circop }}\left(P_{\text {tcop }}\right)=2+0.005\left(P_{\text {tcop }}\right)-A[W] \quad 10 \geq P_{\text {tcop }}(\mathrm{dBm})$

\section{Performance Metrics}

The achievable throughput on the link between the $k$-th coalition and the BS using adaptive modulation and coding is calculated as [6]:

$$
T_{k}\left(\eta_{k}\right)=n_{k}^{R B} k_{s c} \varrho_{s} \varepsilon\left(\eta_{k}\right)[\mathrm{bits} / \mathrm{s}],
$$

where $n_{k}^{R B}$ is the number of resource blocks assigned to the $k$-th coalition, $k_{s c}$ is the number of subcarriers per resource block, $\varrho_{s}$ is the symbol rate per subcarrier, and $\varepsilon\left(\eta_{k}\right)$ is the spectral efficiency for a Long Term Evolution (LTE) system [6].

The user energy efficiency $\beta_{k}$ measures the user throughput per unit of consumed energy. This is based on the total consumed power $P_{\text {total_k}}$, where $P_{\text {total } k} k$ is equal to $P_{\text {tot_simo }}$ when the coalition acts in SIMO mode, and to $P_{\text {tot_mimo }}$ when a virtual MIMO link is constructed.

$$
\beta_{k}=T_{k} / P_{\text {total } \_k}[\text { bits } / \mathrm{J}] .
$$

Additionally, the system energy efficiency $\beta_{\text {sys }}$ is defined as the ratio between the total user throughput and the total power 
spent by all the users in the system:

$$
\beta_{\text {sys }}=\sum_{k=1}^{N} T_{k} / \sum_{k=1}^{N} P_{\text {total_ } k}[\text { bits } / \mathrm{J}] .
$$

\section{Distributed VirTUAL MIMO COALITION FORMATION FRAMEWORK}

In order to find the best RS for an MS to cooperate and forming a virtual MIMO link, we model cooperation with a game theoretic approach derived from the concept of stable marriage with incomplete lists (SMI) [8]. The SMI concept is used to find a stable match between two sets (MSs and RSs). A stable match means that no better alternative pairing exists in the other set, where an MS or RS are better off individually compared with the currently matched elements, thus the SMI leads the system to a stable solution.

In our approach, one set contains $N$ mobiles and the other one contains $R$ relay stations. Furthermore, each single antenna's device preference list consists of a subset of the members of the opposite set ordered by preference. A matching $M$ is a tuple (MS, RS) such that each single antenna device belongs to exactly one tuple. If $(n, r) \in M$, we say that the RS- $r$ is the MS- $n$ 's partner in $M$ and vice versa. The distributed RS selection procedure is as follows:

1) First, each MS in the system sends a broadcast message on the short range (cooperative) link, in order to find the subset of RSs close enough to cooperate with it, which is denoted as $S_{n} \in R$.

2) After the broadcast message, the RSs willing to form a MIMO link share their channel statistics for their reverse link (path loss, shadowing, and channel fading coefficient) and their channel statistics in the cooperative link (path loss) with the subset of mobile stations willing to cooperate with them, which for the RS- $r$ is denoted by $S_{r} \in N$. Mobiles only exchange with the RSs their channel statistics in the reverse link. Hence, each MSs is able to rank its preferred subset of RSs, $S_{n}$, by using the following utility function:

$$
U_{n r}=P_{\text {tot_simo }}-P_{\text {tot_mimo, }} \text {, }
$$

where $U_{n r}$ is computed as the difference between the power spent by the MS- $n$ acting on its own or making a coalition with the RS- $r$. Hence the higher is the utility value, the more the MS- $n$ will be willing to cooperate with the RS- $r$. Additionally, if the utility becomes negative the MS will ignore the RS for coalition formation, since forming a virtual MIMO link will degrade the user's performance. Furthermore, the MS preference list $L_{n}$ is formed by evaluating each element of the subset $S_{n}$ by Eq. (18). MSs that do not obtain any benefit of cooperating with any of the relays in their prefered subset, $S_{n}$, are allowed to act in SIMO mode. As stated in Theorem 1, under certain conditions SIMO may be prefered over MIMO. The RSs need to compute how important or required is their cooperation with the MSs to form a virtual MIMO link. Thus, RSs rank their suitable candidates as follows:

$$
U_{r n}=P_{\text {circ }}\left(\frac{P_{t}\left\|w_{\mathrm{rs}}\right\|^{2}}{M_{t}}\right)-P_{\text {circ }}\left(\frac{P_{t}\left\|w_{\mathrm{ms}}\right\|^{2}}{M_{t}}\right),
$$

where $U_{r n}$ is computed as the difference between the power spent by the RS and the MS when forming a virtual MIMO link. Thus, a high value in the utility means that the RS carries most of the power expenditure for the virtual link, since its channel to the BS experiences better instantaneous conditions than the MS channel. Moreover, the RS' preference list, $L_{r}$, is formed by evaluating each element of $S_{r}$ by Equation (19).

3) After the ranking is done, the SMI procedure given in Algorithm 1, can be applied.

Theorem 1. When the user energy efficiencies becomes a function of the circuit consumed power certain conditions such as: an excessive power expenditure in the cooperative link or favorable transmission conditions in the reverse link will allow a SIMO user to experience a higher energy efficiency performance than a virtual MIMO one. Thus, the following condition is met $\beta_{P_{\text {tot_simo }}} \geq \beta_{P_{\text {tot_mimo }}}$, where $\beta_{P_{\text {tot_simo }}}$ and $\beta_{P_{\text {tot_mimo }}}$ are the user energy efficiencies for a SIMO and MIMO user respectively, which is proven in the Appendix.

Initialization: All MSs must be operating in SIMO mode; while There is an MS- $n$ wanting to form a MIMO link; do $R S_{h}$ is the highest RS in the MS- $n$ preference list, $L_{n}$, to whom it has not yet proposed;

if $R S_{h}$ is free then the MS- $n$ and $R S_{h}$ become engaged; else

$R S_{h}$ is already engaged with an MS- $i$ (where $i \neq n$ ); if $R S_{h}$ prefers the MS-n to the MS-i in its preference list, $L_{r}$; then

MS- $n$ becomes engaged, and the MS- $i$ becomes free;

else $R S_{h}$ remains engaged to the MS- $i$; $R S_{h}$ is deleted from the list of the MS- $n, L_{n}$; end

end Algorithm 1: Stable Marriage with incomplete lists (SMI).

\section{COMParison Schemes And Simulation Scenario}

In order to evaluate our proposal, we describe in this section two distributed coalition formation schemes, which allow the mobiles and relays to cooperate in order to form a virtual MIMO link. Additionally, we present a baseline scheme where all the MSs transmit in SIMO mode. Finally, a centralized global optimum scheme, which is coordinated from the BS, is computed based on an exhaustive search approach: this centralized approach is implemented in order to find the price of anarchy for our proposed scheme. This is computed as the difference in performance between a centralized and a distributed approach. 
TABLE I: Simulation parameters.

\begin{tabular}{cc}
\hline \hline Parameter & Value \\
\hline MSs per macro-cell, $N$ & 20 \\
RSs per macro-cell, $R$ & 120 \\
Number of antennas at the receiver, $M_{r}$ & 2 \\
Intersite-site distance & $700 \mathrm{~m}$ \\
Number of available RBs, $X$ & 20 \\
Number of cells, $D$ & 1 \\
Subcarriers per RB, $k_{s c}$ & 12 \\
Symbol rate per subcarrier, $\varrho_{s}$ & $15 \mathrm{ksps}$ \\
$P_{T x}$ & $31.8 \mathrm{dBm}$ \\
$P_{\text {con }}$ & $23.8 \mathrm{dBm}$ \\
$P_{B B}$ for $\varepsilon=1.1758$ bits & $11.7 \mathrm{dBm}$ \\
Maximum user transmit power & $23 \mathrm{dBm}$ \\
Shadowing, Std. Dev., $\sigma$ & $3 \mathrm{~dB}$ \\
Target SNR, & $3 \mathrm{~dB}$ \\
$\varepsilon$ for $3 \mathrm{~dB}$ SNR & $1.1758 \frac{\mathrm{bits}}{\mathrm{symbol}}$ \\
$\kappa$ & 3.5 \\
\hline
\end{tabular}

\section{A. Minimum Relaying Hop (MRH) Path Loss Selection}

In [9], the authors propose an RS selection method as a function of path loss. Thus, the best RS- $r$ to form a coalition is the one with the least path loss to the MS-n, which is the relay that has the most energy efficient cooperative link:

$$
R S_{c}=\operatorname{argmin}\left\{d_{n r}^{\kappa}\right\},
$$

\section{B. Best Worst (BW) Channel Selection}

In order to form a virtual MIMO link, each MS should take into consideration the quality of the cooperative link, and the RS channel statistics on the reverse link. This is because both have a direct influence on the total energy expenditure to form the virtual MIMO link. In [9], the best worst channel is used in which the relay whose worse channel is the best is selected:

$$
\operatorname{argmin}\left\{\left\|h_{r}, \frac{1}{d_{n r}^{\kappa}}\right\|\right\}
$$

where $h_{r}$ is the fading coefficient between the RS- $r$ and the BS, path loss and shadowing are not considered for the decision making process, since we assume that the other RS candidates are close enough to the MS to experience the same shadowing and path loss.

\section{Simulation scenario}

Monte Carlo simulations are performed using the parameters listed in Table I. This is done in order to compare the performance of our method with the schemes presented above. The simulation is comprised of a single cell with the mobile stations and relays distributed uniformly over the cell. The cell is served by an omnidirectional BS. Furthermore, the system is only noise limited, thus each coalition transmits in an independent RB. In our simulation, we assume that all the coalitions (SIMO or MIMO) try to achieve the same target SNR.

\section{RESULTS}

We generate the cumulative distribution function (CDF), and the graphs that show the distribution of the user circuit consumed power at different distances from the BS for the schemes described in the last section. In Fig. 4, we plot
TABLE II: Percentage of time that the MSs prefer SIMO over MIMO and communication overhead.

\begin{tabular}{ccc}
\hline \hline Method & Percentage & Propagation paths \\
\hline MHR path loss & $0 \%$ & $N \times S_{n}^{*}$ \\
BW channel & $0 \%$ & $2\left(N \times S_{n}^{*}\right)$ \\
SMI & $68 \%$ & $3\left(N \times S_{n}^{*}\right)$ \\
SIMO mode & $100 \%$ & none \\
Centralized global optimum & $66 \%$ & $N+R+(N \times R)$ \\
\hline
\end{tabular}

the user circuit consumed power. We observe, that when the MSs are close to the BS (up to $160 \mathrm{~m}$ ), the circuit power consumption of the SIMO scheme matches the SMI and the global optimum curve. This is because the MSs are able to experience better transmission conditions, when they are close to the BS. Thus, turning on the RF transmitter of the relay stations is less power efficient than transmitting with only one antenna. Furthermore, due to the design of the MS's utility function (18) for the SMI case, MSs are able to switch to SIMO mode when it provides power savings. In the case of the other two distributed approaches (MRH path loss selection and the BW channel selection), both schemes always try to form a virtual MIMO link even if the MSs experience favorable transmission conditions to the BS. Hence, their performance at close distances from the BS is lower than for the other three approaches. Furthermore, in Table II, we show the percentage of time that the MSs in the network prefer SIMO over MIMO transmission. Thus, we see that when the circuit power consumption is considered as an optimization metric and under a set of given conditions, SIMO may be preferred over MIMO. Hence, we verify the results of Theorem 1.

Additionally, in Fig. 5, we show the system energy efficiency. At the $50^{\text {th }}$ percentile, the SMI scheme shows improvements of $37 \%, 42 \%$, and $44 \%$ compared to the SIMO transmission, the MRH path loss and the BW channel respectively. Hence, the proposed SMI approach is more energy efficient than the other two distributed approaches and the baseline case. Furthermore, the price or anarchy for the SMI is $2 \%$, this can be seen as the loss in performance, when the SMI algorithm is compared with the global optimum. Thus, the performance of our distributed approach is similar when compared to a centralized scheme.

Finally, we present in Table II, the channel paths required for each of the proposed methods, where $S_{n}^{*}$ is the number of elements contained in the subset $S_{n}$. The MRH path loss scheme only requires the channel paths between the MSs and the RSs in the cooperative link. Thus, $N \times S_{n}^{*}$ path gains are needed. In the case of the BW channel selection, it requires the paths gains in the cooperative link plus the channel paths between the RSs and the BS, thus the required paths are $2\left(N \times S_{n}^{*}\right)$. For the SMI scheme, we have to add the channel path gains between the MSs and the BS, since MSs exchange their channel statistics with the RSs, hence requiring $3\left(N \times S_{n}^{*}\right)$ channel path gains. The centralized global optimum uses the channel path gains of $N$ MSs, $R$ RSs, and the gains from the cooperative link, $R \times N$ paths. Furthermore, considering the simulation parameters in Table I, the average number of $S_{n}^{*}$ in our simulations is 10 . Hence, for our system 
the SMI requires 600 channel path gains compared with 2540 of the centralized method. Thus, the communication overhead is reduced by $76 \%$.

\section{Vi. Conclusions}

We developed in this paper a distributed game theory algorithm derived from the concept of stable marriage with incomplete lists. Based on the presented results, our approach is a more energy efficient solution with improvements of $42 \%$, $44 \%$, and $37 \%$ when compared to the MRH path loss, the BW channel, and the SIMO mode scheme respectively. The price of anarchy of our proposed method is of $2 \%$. Moreover, the proposed framework reduces the communication overhead of the network by $76 \%$. We have shown analytically and by simulation that when the circuit consumed power is considered for the optimization process and under a given set of conditions, transmitting in SIMO mode is more energy efficient than forming a virtual MIMO link.

\section{APPENDIX}

\section{Proof of Theorem 1}

Equation (16) will be used to prove that under a given set of conditions forming a virtual MIMO link is less energy efficient than transmitting in SIMO mode. Thus, the following condition is met: $\beta_{P_{\text {tot_simo }}} \geq \beta_{P_{\text {tot_mimo }}}$, where $\beta_{P_{\text {tot_simo }}}$ and $\beta_{P_{\text {tot_mimo }}}$ are the user energy efficiencies when transmitting in SIMO and MIMO modes respectively. Furthermore, if the target user rate $T_{k}$ is fixed for both systems, the equation above is equivalent to showing that:

$$
P_{\text {tot_mimo }}\left(P_{t}, P_{\text {tcop }}\right) \geq P_{\text {tot_simo }}\left(P_{t}\right) \text {. }
$$

Additionally, by using Equations (8) and (9), it is easy to see that $P_{\text {tcop }}$ should increase proportionally to the separation distance $d_{n r}$ in order to maintain an acceptable SNR at the RS side. Hence, if $d_{n r} \rightarrow \infty, P_{\text {tcop }} \rightarrow \infty$, and consequently $P_{\text {tot_mimo }}\left(P_{t}, P_{\text {tcop }}\right)$ will dominate over $P_{\text {tot_simo }}\left(P_{t}\right)$.

As stated before, favorable transmission conditions in the reverse link means that the MSs do not need to form a virtual link. Hence, over a long time scale the following is a valid assumption for MIMO and SIMO systems respectively:

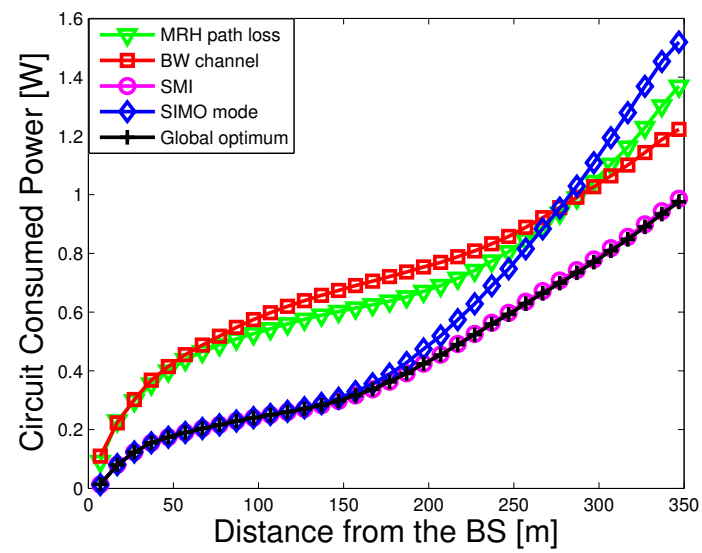

Fig. 4: User circuit consumed power vs distance from the BS.

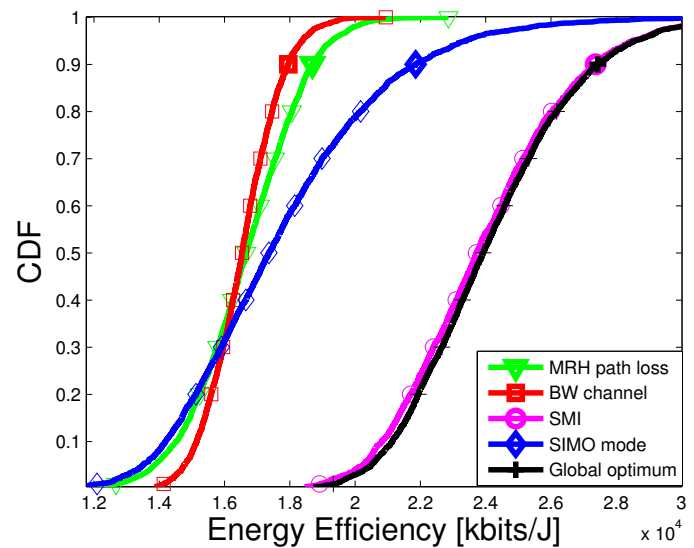

Fig. 5: System CDF energy efficiency.

$\mathbf{E}\left\{\sigma_{\max }^{2}\right\} \leq \mathbf{E}\left\{\|\mathbf{H}\|_{F}^{2}\right\}$, and $\mathbf{E}\left\{\|\mathbf{h}\|_{F}^{2}\right\}=M_{r}$, where $\mathbf{E}\left\{\|\mathbf{H}\|_{F}^{2}\right\}=M_{t} M_{r}$ [1]. Thus, by using Equations (6) and (7) the average SNR E $\left\{\eta_{k}\right\}$ for a MIMO and SIMO coalition can be computed as follows: $\mathbf{E}\left\{\eta_{k_{-} \text {mimo }}\right\} \leq \frac{P_{r} M_{r} M_{t}}{\rho}$, and $\mathbf{E}\left\{\eta_{k_{-} \text {simo }}\right\}=\frac{P_{r} M_{r}}{\rho}$. Hence, in order to achieve the same average SNR at the BS side, a SIMO coalition consumes approximately $M_{t}$ times more transmitted power than a MIMO coalition, thus, $P_{t_{-} \text {mimo }} \times M_{t} \approx P_{t_{-} \text {simo. }}$. Furthermore, make $P_{t_{-} \text {mimo }} \times M_{t}$ and $P_{t_{-} \text {mimo }}$ the inputs for the uplink transmit power in Equations (10) and (11) respectively. Making $P_{t_{-} \text {mimo }}$ to tend to a small value implies the MSs experience favorable transmission conditions in the reverse link, it is easy to see in Equation (12) that the reverse link power that is spent when a virtual MIMO link is formed will cause $P_{\text {tot_mimo }}\left(P_{t}, P_{\text {tcop }}\right)$ to be higher than $P_{\text {tot_simo }}\left(P_{t}\right)$, for favorable transmission conditions in the uplink.

\section{REFERENCES}

[1] A. Paulraj, R. Nabar, and D. Gore, Introduction to space-time wireless communications, C. U. Press, Ed. Cambridge University Press, Jul. 2003.

[2] J. Jiang, M. Dianati, M. Imran, and Y. Chen, "Energy efficiency and optimal power allocation in virtual-MIMO systems," in Proceedings of the IEEE 76th Vehicular Technology Conference, 2012, pp. 1-6.

[3] S. Sesia, I. Toufik, and M. Bake, LTE-The UMTS Long Term Evolution From Theory to Practice, 1st ed., C. Wiley, Ed. Wiley, Chichester, 2009.

[4] W. Saad, Z. Han, and M. Debbah, "A distributed coalition formation framework for fair user cooperation in wireless networks," IEEE Transactions on Wireless Communications, vol. 8, no. 9, pp. 4580-4593, 2009.

[5] A. Jensen et al., "LTE UE power consumption model for system level energy and performance optimization," in Proceedings of the IEEE 76th Vehicular Technology Conference, 2012, pp. 1-6.

[6] H. Burchardt et al., "Uplink interference protection and scheduling for energy efficient OFDMA networks," EURASIP Journal on Wireless Communications and Networks, vol. 2012, no. 180, p. 19, 2012.

[7] K. Bobae, K. Cholho, and L. Jongsoo, "A dual-mode power amplifier with on-chip switch bias control circuits for LTE handsets," IEEE Transactions on Circuits and Systems, vol. 58, no. 12, pp. 857-861, 2011.

[8] K. Iwama et al., "A survey of the stable marriage problem and its variants," in Proceedings of the International Conference on Informatics Research for Development of Knowledge Society Infrastructure, Jan 2008, pp. 131-136.

[9] V. Sreng et al., "Relayer selection strategies in cellular networks with peer-to-peer relaying," in Proceedings of the IEEE 58th Vehicular Technology Conference, Oct. 2003, pp. 1949-1953. 